\title{
Implied Liquidity Risk Premia in Option Markets
}

\author{
Florence Guillaume*, Gero Junikeł Peter Leoni; Wim Schoutens ${ }^{\S}$
}

August 22, 2018

\begin{abstract}
The theory of conic finance replaces the classical one-price model by a two-price model by determining bid and ask prices for future terminal cash flows in a consistent manner. In this framework, we derive closedform solutions for bid and ask prices of plain vanilla European options, when the density of the log-returns is log-concave.

Assuming that log-returns are normally or Laplace distributed, we apply the results to a time-series of real market data and compute an implied liquidity risk premium to describe the bid-ask spread. We compare this approach to the classical attempt of describing the spread by quoting Black-Scholes implied bid and ask volatilities and demonstrate that the new approach characterize liquidity over time significantly better.

Key-Words: Conic finance, distortion functions, WANG-transform, Laplace distortion
\end{abstract}

\section{Introduction}

The risk taken by an investor by holding an asset with an uncertain future cash flow can be described by coherent risk measures, which have been introduced axiomatically by Artzner et al. (1999). A coherent risk measure maps the set of bounded random variables to the real numbers fulfilling four axioms: cash invariance, monotonicity, sub-additivity, and positive homogeneity. An important subclass of risk measures is described by distortion functions, which are concave functions mapping the unit interval into the unit interval and have been studied in a general context by Kusuoka (2001). For example the expected shortfall can be represented by distortion functions. Under a distortion function,

\footnotetext{
*University of Antwerp, Middelheimlaan 1, 2020 Antwerpen, Belgium. Email: Florence.Guillaume@uantwerpen.be.

${ }^{\dagger}$ Department of Mathematics, Universitat Autònoma de Barcelona, Building C Science Faculty, 08193 Bellaterra (Barcelona), Spain. Email: junike@mat.uab.cat. This research is partially supported by 13 th UAB-PIF scholarship.

‡Electrabel NV/SA, Regentlaan 8, Boulevard du Régent 8, 1000 Brussels, Belgium. Email: peter.leoni@kuleuven.be.

$\S$ Department of Mathematics, KU Leuven, Celestijnenlaan 200 B, 3001 Leuven, Belgium. Email: wim.schoutens@kuleuven.be.
} 
the risk of a random cash flow $X$ is calculated as its distorted expectation by reweighting the distribution function of $X$ : the probability of losses gets higher weight while the probability of gains is lower weighted.

Distortion functions play an important role in finance: beside their applications in risk-measurement, they have also been used by Cherny and Madan (2009) in order to define a new class of performance measures, the so called acceptability indices. An acceptability index represents the attractiveness of a cash flow, a famous example being the gain-loss ratio, see Bernardo and Ledoit (2000). There is a strong duality between an acceptability index and risk measures: any unbounded acceptability index can be represented by a family of risk measures. Madan and Cherny (2010) applied the theory of performance measures and developed the theory of conic finance which allows prices to depend on the direction of the trade: there are two prices: an ask price for buying an asset from the market and a usually smaller bid price for selling the asset to the market. They defined the bid price of a cash flow $X$ by its discounted distorted expectation and the ask price by minus the discounted distorted expectation of the cash flow $-X$. Cherny and Madan considered four families of concave distortion functions: MINVAR, MAXVAR, MINMAXVAR and MAXMINVAR, which all depend on some parameter $\gamma \geq 0$ describing the liquidity of the market. The greater $\gamma$, the greater the bid-ask spread and the less liquid the market. Given real market data, it is possible to compute an implied $\gamma$ matching the bid and ask prices exactly. Such a $\gamma$ is called implied liquidity risk premium.

Another popular example of a concave distortion function used in actuarial science, the so called Wang-transform, was proposed by Wang (2000). It involves the standard cumulative normal distribution $\Phi$ and its inverse. For some level $\gamma \geq 0$, Wang defined a distortion function by

$$
\Psi_{\mathrm{WANG}}^{\gamma}(u)=\Phi\left(\Phi^{-1}(u)+\gamma\right), u \in[0,1], \quad \gamma \geq 0 .
$$

The WANG-transform is a family of distortion functions on the index set $\gamma \geq 0$. For $\gamma=0$, the WANG-transform is the identity function, there is in fact no distortion and the distorted expectation is equal to the usual expectation. For an increasing $\gamma$, the distortion is more and more severe and in the limit for $\gamma \rightarrow \infty$, the distorted expectation corresponds to the worst case risk measure and is equal to the maximum possible loss of the cash flow.

The WANG-transform has been modified by Wang (2002) to a two factor model replacing the normal distribution in Equation (1) by Student's tdistribution but leaving the inverse normal distribution inside the brackets untouched. Kijima and Muromachi (2006) introduced a new transform involving a non-central t-distribution and the inverse of a standard t-distribution. The classical WANG-transform has been extended to the multidimensional case by Kijima (2006). Kijima and Muromachi (2008) generalized the WANG-transform and constructed a transformation using the normal distribution and the inverse of the cumulative distribution function of the quotient of a normally distributed random variable and some independent positive random variable $Y$. For $Y=1$ the classical WANG-transform is obtained. Tsukahara (2009) generalized the 
WANG-transform by replacing the normal distribution function $\Phi$ in Equation (1) by a general distribution function $F$ and its inverse by $F^{-1}$. Tsukahara called such a distribution function a one-parameter distortion group and showed the distortion function is concave, if $F$ is $\log$ concave. We say such a distortion function is induced by the distribution function $F$.

In this article, we apply the results of Tsukahara (2009) to conic finance: Let us assume that the log-returns of some random cash flow have distribution $F$ and that $F$ is $\log$ concave. If we use the family of distortion functions that is induced by $F$, we are able to derive explicit formulas for the bid and ask prices of the cash flow. In particular the bid and ask prices of European vanilla options are equal to the risk-neutral price of an option on the underlying with an adjusted dividend yield. It is then possible to derive closed-form solutions provided that the log-returns are normally or Laplace distributed.

This article is structured as follows: In Section 2 we introduce distortion functions and recall an important connection to distribution functions. The result is applied to conic finance in Section 3 to derive close-form solutions for bid and ask prices of European options. Two important examples, the BlackScholes and the Laplace-model are discussed. In Section 3.4 the concept of the implied liquidity risk premium is defined and applied to real data in Section 3.5. Given bid and ask market prices, an implied liquidity risk premium $\gamma \geq 0$ can be computed such that model bid and ask prices are equal to market prices, compare with Corcuera et al. (2012). We do this for both the Black-Scholes and the Laplace-model and compare the new approach to the commonly used approach by practitioners of quoting implied bid and ask volatilities to describe the bid-ask spread.

\section{Some Results on Distortion Functions}

Madan and Cherny (2010) defined bid and ask prices via distorted expectations that are based on distortion functions. Let us recall the definition of distortion functions. A comprehensive introduction of risk measures based on concave distortion functions can be found e.g. in Föllmer and Schied (2011, Section 4.6).

Definition 2.1. (Family of distortion functions). A family of distortion functions $\left(\Psi^{\gamma}\right)_{\gamma \geq 0}$ is a set of functions $\Psi^{\gamma}:[0,1] \rightarrow[0,1]$ that are monotonically increasing, continuous and concave for all $\gamma \geq 0$ and for which $\Psi^{\gamma}(0)=0$ and $\Psi^{\gamma}(1)=1$. Moreover the family is monotonically increasing and continuous at $\gamma$, i.e. it holds that for all $u \in[0,1]: \Psi^{\gamma_{1}}(u) \leq \Psi^{\gamma_{2}}(u)$ for $\gamma_{1} \leq \gamma_{2}$.

In what follows, we will assume that $\Psi^{0}(u)=u, u \in[0,1]$. Let $F$ be a distribution function with density $f$. We say a family of distortion functions $\left(\Psi^{\gamma}\right)$, satisfying the requirements of Definition 2.1, is induced by $F$, if it holds

$$
\Psi^{\gamma}(u)=F\left(F^{-1}(u)+\gamma\right), u \in[0,1], \quad \gamma \geq 0,
$$


where $F^{-1}$ is understood to be the generalized inverse of $F$ and we define $F(-\infty)=0$ and $F(\infty)=1$.

Remark 2.2. The function $\Psi^{\gamma}$ defined by Equation (2) is continuous in $u$ if $F$ and $F^{-1}$ are continuous. Tsukahara (2009) showed that $\Psi^{\gamma}$ is concave, if $F^{\prime \prime} / F^{\prime}$ is decreasing: such densities are called log-concave densities. There exists a concave function $\varphi$ such that $f(x)=\exp (\varphi(x))$. The log-concave densities used in this article to describe log-returns are the normal and the Laplace distribution.

The most prominent example of a family of distortion functions induced by a distribution function, is the WANG-transform, which is induced by the normal distribution, compare with Equation (1). We provide another example:

Example 2.3. A random variable with mean zero and variance one which is Laplace distributed, has the following distribution function

$$
F(x)= \begin{cases}\frac{1}{2} e^{\sqrt{2} x} & , x<0 \\ 1-\frac{1}{2} e^{-\sqrt{2} x} & , x \geq 0 .\end{cases}
$$

It induces via Equation (2) the Laplace distortion

$$
\Psi_{\text {Laplace }}^{\gamma}(u)= \begin{cases}u e^{\sqrt{2} \gamma} & , u \in\left[0,1 / 2 e^{-\sqrt{2} \gamma}\right) \\ 1-\frac{e^{-\sqrt{2} \gamma}}{4 u} & , u \in\left[1 / 2 e^{-\sqrt{2} \gamma}, 1 / 2\right) \\ u e^{-\sqrt{2} \gamma}+1-e^{-\sqrt{2} \gamma} & , u \in[1 / 2,1],\end{cases}
$$

which is linear for $u<1 / 2 e^{-\sqrt{2} \gamma}$ and for $u \geq 1 / 2$. In between it behaves like a reciprocal function and it is clearly concave. Applying the Laplace distortion to a uniform distribution function, which appears e.g. via a Monte Carlo simulation, leads to a new interesting interpretation of the parameter $\gamma$. In Figure 1 the density of a Laplace distorted uniform distribution is drawn. It is high and constant at the beginning, then it drops sharply and is quite low and constant at the right hand side of the median. So if we only wish to distort the $q$-quantile of a uniform distribution strongly, we can simply choose $\gamma=-\frac{1}{\sqrt{2}} \log (2 q)$. On the other hand, if real data is given and we calculate implicitly the parameter $\gamma$, the value $\frac{1}{2} e^{-\sqrt{2} \gamma}$ can be interpreted as the quantile that is the most strongly distorted.

In general, it is desirable that

$$
\lim _{u \searrow 0} \Psi_{\gamma}^{\prime}(u)=\infty, \gamma>0
$$

and

$$
\lim _{u \nearrow 1} \Psi_{\gamma}^{\prime}(u)=0, \quad \gamma>0,
$$

where we assumed that the distortion function is differentiable and we where we defined $\Psi_{\gamma}^{\prime}(u)=\frac{\partial}{\partial u} \Psi^{\gamma}(u)$, compare with Cherny and Madan (2009) and Balbás 


\section{Density of Laplace Distorted Uniform Distribution}

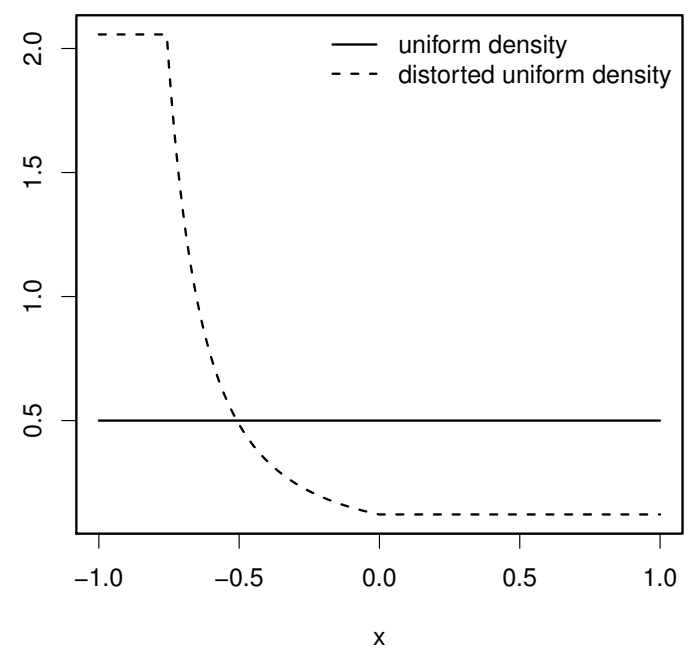

Figure 1: Density of Laplace distorted uniform distribution on $[-1,1]$ at distortion level $\gamma=1$.

et al. (2009). High losses happen somewhere on the left of a distribution function: for $x \rightarrow-\infty$ it holds $F_{X}(x) \searrow 0$. Hence, Equation (3) ensures loss aversion. On the other hand, big gains happen somewhere on the right: for $x \rightarrow \infty$ it follows $F_{X}(x) \nearrow 1$, thus Equation (4) ensures against being enticed by large gains.

It is well known that the WANG transform satisfies Equations (3) and (4), see for example Madan and Schoutens (2016). Example 2.3 shows that the Laplace distortion does not satisfy Equation (3) neither (4).

\section{Applications to Conic Finance}

The aim of this section is to derive analytic formulas for bid and ask prices of European options. We will see that under certain conditions, the bid (ask) price of a call option on a stock with dividend yield $q$ can be calculated as the risk-neutral price of an option with the same strike and maturity but an increased (decreased) dividend yield $\tilde{q}$. The key idea is to use a family of distortion functions which is induced by the distribution function of the log-returns. Then the computation of the distorted integrals is straightforward and leads to analytic formulas for bid and ask prices in case of the normal and the Laplace distribution. 


\subsection{Stock model}

Inspired by Corcuera et al. (2009), let us model a stock at some terminal date $T$ by a random variable $S_{T}$, which is defined in the following way: let $Z$ be a random variable with mean zero and variance equal to 1 . Its distribution function is denoted by $F_{Z}$, its density by $f_{Z}$. The random variable $\sqrt{T} Z$ has then variance $T$ and the underlying $S_{T}$ at time $T$ is defined by

$$
S_{T}=S_{0} e^{(r-q+\omega) T+\sigma \sqrt{T} Z}
$$

where $\sigma>0, r$ is the risk-free rate, $q$ the dividend yield and $\omega \in \mathbb{R}$ is a mean correcting term, i.e. $\omega$ is chosen such that

$$
e^{-(r-q) T} E\left(S_{T}\right)=S_{0},
$$

where the expectation is taken under a risk-neutral measure $\mathbb{Q}$. In the following, we assume that $F_{Z}$ is symmetric about zero, i.e. $F_{Z}(-x)=1-F_{Z}(x)$ for all $x \in \mathbb{R}$.

\subsection{Conic Bid and Ask prices}

We then introduce a terminal cash flow $X$, for example a call option on $S_{T}$, and denote by $F_{X}$ the distribution function of $X$ with respect to the measure $\mathbb{Q}$. By Madan and Cherny (2010), the bid and ask prices of the random cash-flow $X$, without hedging opportunities, are defined as distorted expectations, with respect to some $\gamma \geq 0$. For the bid price, we have that

$$
\begin{aligned}
\operatorname{bid}^{\gamma}(X) & =e^{-r T} \int_{-\infty}^{\infty} x d \Psi^{\gamma}\left(F_{X}(x)\right) \\
& =e^{-r T} \int_{-\infty}^{\infty} x \Psi_{\gamma}^{\prime}\left(F_{X}(x)\right) f_{X}(x) d x,
\end{aligned}
$$

where $\left(\Psi^{\gamma}\right)_{\gamma>0}$ is a family of distortion functions. For the second equation, we assume that the distribution function $F_{X}$ of $X$ is differentiable with density $f_{X}$. Similarly, the ask price is defined by

$$
\operatorname{ask}^{\gamma}(X)=-e^{-r T} \int_{-\infty}^{\infty} x d \Psi_{\gamma}\left(F_{-X}(x)\right) .
$$

If $\gamma=0$, the bid and ask prices coincide and are equal to the risk-neutral price of the cash flow $X$, which is the discounted expectation of $X$ under the risk-neutral measure $\mathbb{Q}$.

\subsection{Bid and Ask prices of European Options}

We now introduce a call option with strike $K$ and maturity $T$ on the underlying $S_{T}$. It is easy to see that the distribution of the call option $C=\left(S_{T}-K\right)^{+}$is

$$
\begin{aligned}
F_{C}(x) & =F_{S_{T}}(x+K), x \geq 0 \\
& =F_{Z}\left(\frac{\log (x+K)-\log \left(S_{0}\right)-(r-q+\omega) T}{\sigma \sqrt{T}}\right), x \geq 0 .
\end{aligned}
$$


Let us assume that $F_{Z}$ induces a family of distortion functions by

$$
\Psi_{Z}^{\gamma}(u)=F_{Z}\left(F_{Z}^{-1}(u)+\gamma\right), \gamma \geq 0,
$$

compare with Remark 2.2. In particular, we assume that $f_{Z}$ belongs to the family of log-concave densities. This will lead to particularly simple formulas for the bid and ask prices because the distorted distribution function can then explicitly be calculated via Equations (7) and (9), e.g. for a call option, it holds

$$
\Psi_{Z}^{\gamma}\left(F_{C}(x)\right)=F_{Z}\left(\frac{\log (x+K)-\log \left(S_{0}\right)-(r-q+\omega) T}{\sigma \sqrt{T}}+\gamma\right), x \geq 0 .
$$

We calculate the bid price of a call option by

$$
\begin{aligned}
\operatorname{bid}^{\gamma}(C) & =e^{-r T} \int_{-\infty}^{\infty} x d \Psi_{Z}^{\gamma}\left(F_{C}(x)\right) \\
& =e^{-r T} \int_{0}^{\infty} x \frac{f_{Z}\left(\frac{\log (x+K)-\log \left(S_{0}\right)-(r-q+\omega) T}{\sigma \sqrt{T}}+\gamma\right)}{\sigma \sqrt{T}(x+K)} d x \\
& =e^{-r T} \int_{-d+\gamma}^{\infty}\left(S_{0} e^{\sigma \sqrt{T} y+(r-q+\omega) T-\sigma \sqrt{T} \gamma}-K\right) f_{Z}(y) d y
\end{aligned}
$$

where

$$
d=\frac{\log \left(\frac{S_{0}}{K}\right)+(r-q+\omega) T}{\sigma \sqrt{T}} .
$$

From Equation (10) we see that the bid price of an option $C$ on a stock with dividend yield $q$ at level $\gamma \geq 0$ equals the risk neutral price of an option on a stock with a different dividend yield $\tilde{q}=q+\frac{\gamma \sigma}{\sqrt{T}}$.

Similarly, the ask price can be obtained by evaluating $F_{-C}$, it holds

$$
\begin{aligned}
\operatorname{ask}^{\gamma}(C) & =e^{-r T} \int_{-d-\gamma}^{\infty}\left(S_{0} e^{\sigma \sqrt{T} y+(r-q+\omega) T+\sigma \sqrt{T} \gamma}-K\right) f_{Z}(y) d y \\
& =\operatorname{bid}^{-\gamma}(C)
\end{aligned}
$$

Hence, if we have an analytic formula for the bid price, we just need to substitute $\gamma$ by $-\gamma$ to get an analytic formula for the ask price.

Analogically, it holds for the bid price of an European Put option $P=$ $\left(K-S_{T}\right)^{+}$

$$
\operatorname{bid}^{\gamma}(P)=e^{-r T} \int_{d+\gamma}^{\infty}\left(K-S_{0} e^{-\sigma \sqrt{T} y+(r-q+\omega) T+\sigma \sqrt{T} \gamma}\right) f_{Z}(y) d y
$$

and the ask price of a put option can be expressed by

$$
\begin{aligned}
\operatorname{ask}^{\gamma}(P) & =e^{-r T} \int_{d-\gamma}^{\infty}\left(K-S_{0} e^{-\sigma \sqrt{T} y+(r-q+\omega) T-\sigma \sqrt{T} \gamma}\right) f_{Z}(y) d y \\
& =\operatorname{bid}^{-\gamma}(P)
\end{aligned}
$$


Summarizing, the bid price of a call option and the ask price of a put option are equal to the risk-neutral prices of a call and a put option respectively, replacing the dividend yield $q$ of the stock by $q+\frac{\sigma \gamma}{\sqrt{T}}$. The ask price of a call option and the bid price of a put option are equal to risk-neutral price of a call and a put option respectively, replacing the dividend yield by $q-\frac{\sigma \gamma}{\sqrt{T}}$.

We will provide two examples where Equation (10) can be calculated explicitly.

Example 3.1. As already mentioned by Madan and Schoutens (2016, Example 5.5), assuming a Black-Scholes setting, i.e. $Z$ is standard normally distributed with distribution function $\Phi$ and $\omega=-\frac{1}{2} \sigma^{2}$ and using the WANG-transform, leads to the following formulas for the bid price of a call option and a put option

$$
\begin{aligned}
& \operatorname{bid}_{\mathrm{WANG}}(C)=S_{0} e^{-\left(q+\frac{\gamma \sigma}{\sqrt{T}}\right) T} \Phi\left(d_{1}-\gamma\right)-e^{-r T} K \Phi\left(d_{2}-\gamma\right) \\
& \operatorname{bid}_{\mathrm{WANG}}(P)=e^{-r T} K \Phi\left(-d_{2}-\gamma\right)-S_{0} e^{-\left(q-\frac{\gamma \sigma}{\sqrt{T}}\right) T} \Phi\left(-d_{1}-\gamma\right)
\end{aligned}
$$

where

$$
d_{1}=\frac{\log \left(S_{0} / K\right)+\left(r-q+\sigma^{2} / 2\right) T}{\sigma \sqrt{T}}
$$

and $d_{2}=d_{1}-\sigma \sqrt{T}$ are defined as in the classical Black-Scholes model. The ask prices are equal to the bid prices, replacing $\gamma$ by $-\gamma$. For $\gamma=0$, we obtain the classical Black-Scholes formula.

The Laplace distribution is particularly interesting because mathematically it is even easier to handle than the normal distribution and it has fatter tails. While the logarithm of the density of the normal distribution decays quadratically, the logarithm of the Laplace density decreases linearly. Thus using the Laplace distribution instead of the normal distribution can overcome some of the criticism of the Black-Scholes model.

Example 3.2. Let $T>0$ and let $Z$ be Laplace distributed with mean zero and variance 1 . In particular, $Z$ has density

$$
f_{Z}(x)=\frac{1}{\sqrt{2}} e^{-\sqrt{2}|x|} .
$$

Let us use the Laplace distortion as defined in Example 2.3 and assume $\sigma^{2} T<2$ and let $\omega=\frac{1}{T} \log \left(1-\frac{1}{2} \sigma^{2} T\right)$, which makes the underlying in Equation (5) risk neutral. See also Madan (2016) for the use of the Laplace distribution in pricing European options. Note that the integral in Equation (10) is infinite if $\sigma^{2} T \geq 2$, independently of the choice of $\omega$. We should not worry to much about this: from a practical point of view, the maturity $T$ and volatility $\sigma$ usually do not exceed the limit, i.e. $\sigma^{2} T<2$. E.g. if we look at a time-horizon of less then eight years and a yearly volatility of $50 \%$ or less, we are well below the limit. From a mathematical point of view, we know by Equation (6) that the expectation of $S_{T}$ under the risk neutral measure must be finite, in particular it holds

$$
E\left(S_{T}\right)<\infty
$$




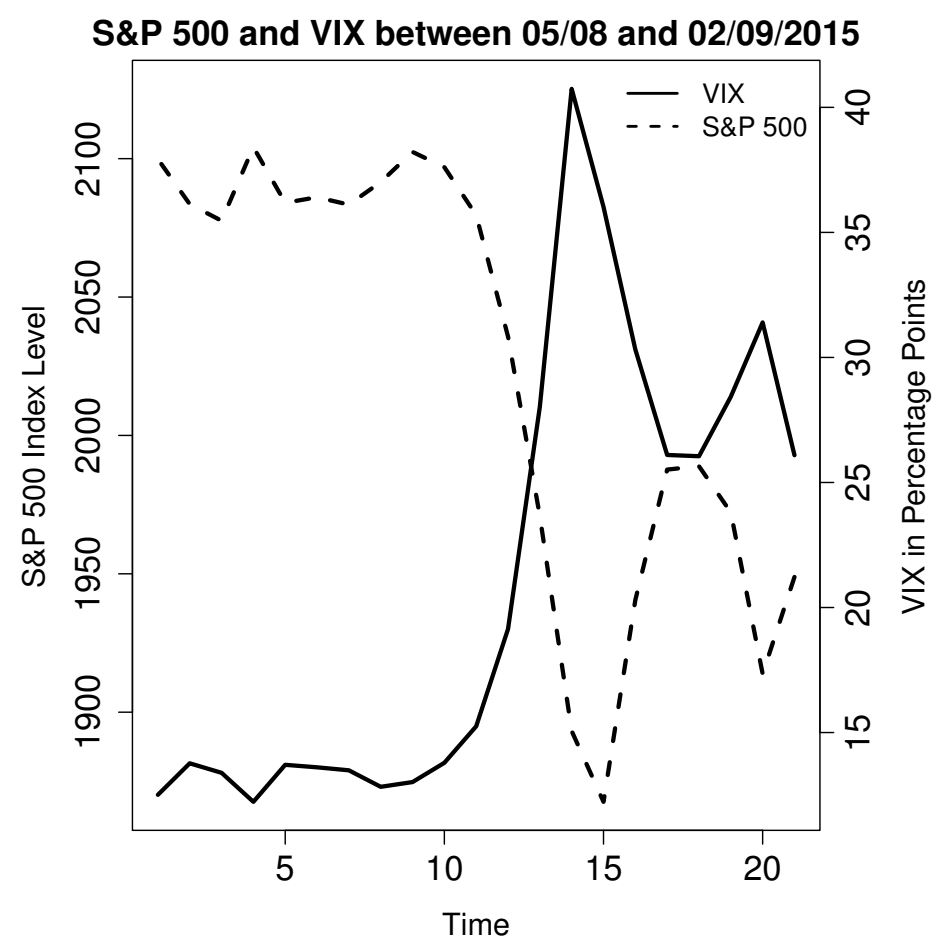

Figure 2: S\&P 500 and VIX between August, 5th and September, 2nd 2015.

This is equivalent to $E\left(e^{\sigma \sqrt{T} Z}\right)<\infty$. On the other hand it holds

$$
E\left(e^{\sigma \sqrt{T} Z}\right)=\int_{\mathbb{R}} e^{\sigma x} \frac{1}{\sqrt{2 T}} e^{-\frac{\sqrt{2}}{\sqrt{T}}|x|} d x= \begin{cases}\infty & , \sigma \sqrt{T} \geq \sqrt{2} \\ \frac{2}{2-\sigma^{2} T} & , \sigma \sqrt{T}<\sqrt{2} .\end{cases}
$$

Therefore $\sigma^{2} T<2$ must hold but as $\frac{2}{2-\sigma^{2} T} \rightarrow \infty$ for $\sigma^{2} T \nearrow 2$, the integral may be arbitrary large. Closed-form solutions for bid and ask prices of European options can be obtained by taking the corresponding closed-form solutions in Madan (2016, Section 2.1) and replacing $q$ by $q+\frac{\gamma \sigma}{\sqrt{T}}$, respectively by $q-\frac{\gamma \sigma}{\sqrt{T}}$.

\subsection{Implied Liquidity Risk Premium (ILRP)}

The concept of implied liquidity has been introduced by Corcuera et al. (2012), Dhaene et al. (2012) and Albrecher et al. (2013). It is similar to the idea of implied volatility and computes implicitly two parameters $\gamma_{b}$ and $\gamma_{a}$ such that modelled bid and ask prices match real market prices.

Given some real market data of bid and ask prices of a cash-flow $X$, we assume that the risk-neutral measure $\mathbb{Q}$ is chosen, such that the mid price is equal 


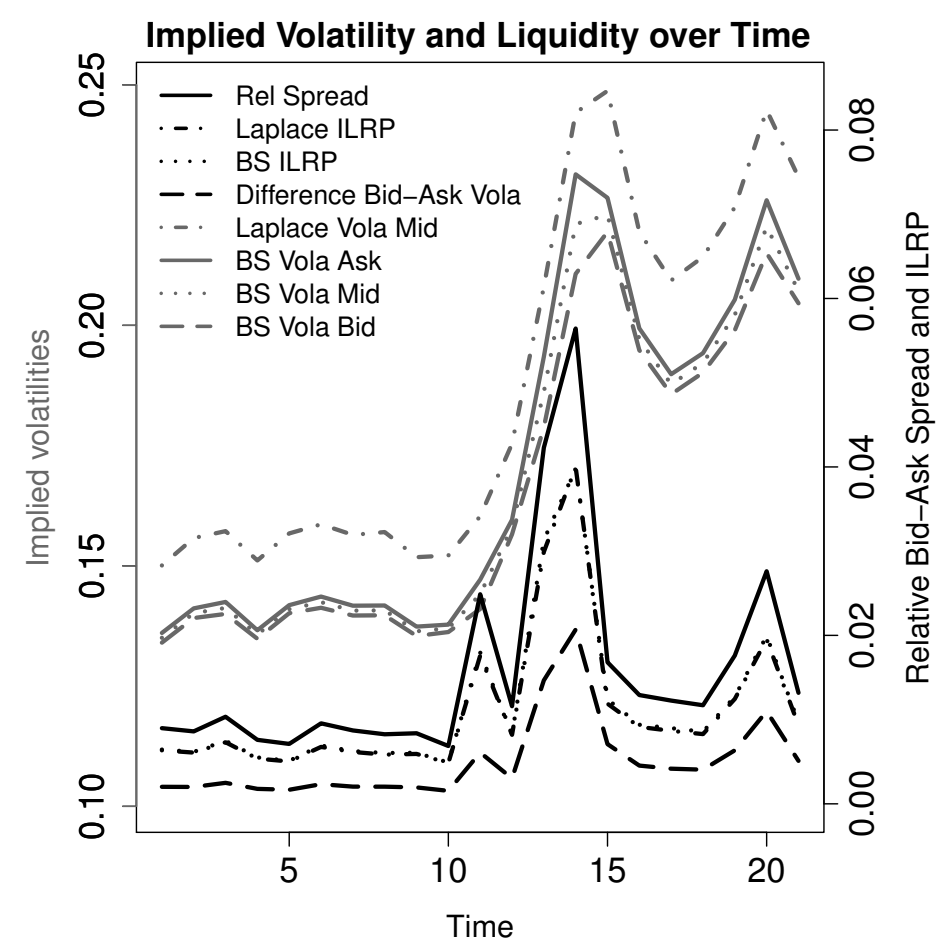

Figure 3: Average implied volatility and liquidity over time of ATM-Call options with maturity varying between 0.42 and 0.55 years and moneyness ranging between 0.98 and 1.02 .

to the discounted expectation of $X$ under $\mathbb{Q}$. For example if $X$ is an European option, and the underlying is described by the Black-Scholes model, one would compute an implied volatility such that the Black-Scholes price matches the given mid price of the option. Thus we assume the distribution $F_{X}$ is known and call a non-negative number $\gamma_{b}$ such that $\operatorname{bid}^{\gamma_{b}}(X)$, defined in Equation (7), exactly matches the given market bid-price as the implied liquidity risk premium at the bid-side. We similarly define $\gamma_{a} \geq 0$ such that $\operatorname{ask}^{\gamma_{a}}(X)$ is equal to the given ask price as the implied liquidity risk premium at the ask-side. The pair $\left(\gamma_{b}, \gamma_{a}\right)$ is simply called the implied liquidity risk premium (ILRP).

We saw in the Section 3.3, that bid and ask prices of European options on a stock with dividend yield $q$ at level $\gamma \geq 0$ equal the risk neutral price of the same option, but on a stock with an adjusted dividend yield $\tilde{q}=q \pm \frac{\gamma \sigma}{\sqrt{T}}$. Bid and ask prices can thus be described by drift adjustments, hence the name liquidity risk premium. 


\subsection{Application to real Market Data}

We apply both the Black-Scholes model and the Laplace-model from Example 3.1 and 3.2 to bid and ask prices of real option data and compute the ILRP. For a time-series of 21 days, between August, 5th and September, 2nd 2015, we look at 1820 end-of-day bid and ask prices of European plain vanilla call and put options on the S\&P 500 with maturities ranging from about 0.42 to 2.36 years and moneyness between 0.83 and 1.09. The options are obtained from the Chicago Board Options Exchange.

As shown in Figure 2, the uncertainty of Standard \& Poor's 500 stock market index rose sharply during that period. On August, 24th, which was termed "Black Monday" by China's media due to the China's stock market crash, the CBOE Volatility Index (VIX) reached 53.29 points during the day and closed at 40.74 points. Only a week before, on August, 17th, the VIX closed at 13.02 points. It is well known that liquidity of stock markets usually drops, when uncertainty rises. Indeed, while at the beginning of the time-series, the relative bid-ask spread is less than $1 \%$ for at-the-money call options with maturity of about half a year, it rises to more than $5.6 \%$ on August, 24th for the same type of options. In the following, we are going to compare the relative bid-ask spread, the ILRP and the difference between implied bid and ask volatilities.

We compute for each option at each timepoint the Black-Scholes implied volatility $\sigma_{\text {Mid }}^{\mathrm{BS}}$ and Laplacian implied volatility $\sigma_{\text {Mid }}^{\mathrm{L}}$ matching exactly the midprice using the classical Black-Scholes formula and the formulas derived by Madan (2016, Section 2.1) for the Laplace-model, i.e. the formulas in Equations (12) and (14) setting $\gamma=0$. We get a typical volatility smile for both models, even though the Laplacian implied volatility surface is slightly flatter than the Black-Scholes implied volatility surface.

For each option, at each timepoint and for both models, we use the mid-price implied volatility as estimate of the volatility in Equation (5) and compute the ILRP, $\left(\gamma_{b}, \gamma_{a}\right) \in \mathbb{R}_{+}$, such that the model bid and ask prices match exactly the quoted market bid and ask prices, see Section 3.4. For example, the BlackScholes bid-price in Equation (15), is equal to the quoted market bid price of a call option, when using the implied $\gamma_{b}$ and $\sigma_{\text {Mid }}^{\text {BS }}$ as input parameters. Note that for most options $\gamma_{b}$ and $\gamma_{a}$ are almost identical, only for very deep out-of-the money options the difference between both values is more pronounced.

In industry, traders usually prefer quoting the implied volatility instead of the mid-price, because the implied volatility is comparable across strikes, maturities and underlying assets. With the same argument, it seems more appropriate to quote the bid-ask spread in terms of the ILRP because spreads behave in a non-linear way across strikes, maturities and underlyings while the ILRP improves comparability across all three dimensions.

So far traders quote implied bid and ask volatilities and describe the bid-ask spread implicitly by the difference of the implied bid and ask volatilities. This procedure needs to be compared to the approach to describe the bid-ask spread by the ILRP. Note that while for some options it is not possible to compute the implied bid volatility, because the bid-price is below the arbitrage-free price, 
for all options there exists an implied $\gamma_{b}$ matching the bid-price exactly. We removed all options from the data-set where it is not possible to compute an implied bid volatility.

In Figure 3, the time-series of mean values $\frac{\gamma_{a}+\gamma_{b}}{2}$ for the Black-Scholes model and the Laplace-model are shown for at-the-money call options with maturity of about half a year and are compared to the relative bid-ask spread and the implied bid-ask volatilities over time. While the relative bid-ask spread rose from timepoint 12 (August, 20th) to timepoint 14 (August 24th) from 1.2\% to 5.6\%, hence by the factor 4.87 , the ILRP make a similar move and rose by the factor 4.81. But the difference between bid and ask implied volatilities changed by the factor 6.85. Hence describing the bid-ask spread by quoting implied bid and ask volatilities, overestimates the change in liquidity by about $35 \%$. Looking at put options instead or analysing options with different maturities or moneyness levels, gives a similar picture. Figure 4 illustrates the relative difference of four liquidity measures, respectively between two successive timepoints. The relative bid-ask spread, the Black-Scholes and the Laplacian ILRP and the difference between bid and ask Black-Scholes implied volatilities are compared for at-themoney and out-of-the-money call and put options with maturities of about half a year. It is not unusual that quoting the bid-ask spread using implied volatilities overestimates an up or down move in liquidity by $40 \%$ and more compared to the relative bid-ask spread. Only for in-the-money options, all four liquidity measures behave similarly.

The correlation between the relative bid-ask spread and $\gamma_{b}$ or $\gamma_{a}$ for the different maturities and option types (call and put), lay between 0.91 and 0.99 for the Black-Scholes and the Laplace-model. That makes the ILRP a more intuitive measure for liquidity than quoting the spread implicitly by stating implied volatilities for both bid and ask prices.

\section{Summary}

In a static conic finance framework, bid and ask prices of a future random cash flow are defined as distorted expectations of the cash flow using some family of distortion functions. We have seen that bid and ask prices of European options can be calculated as the risk neutral price of the same option but on an underlying with an adjusted dividend yield by adequately choosing the family of distortion functions. In particular, the bid price of a call option with maturity $T$ on a stock with dividend yield $q$ at liquidity risk premium $\gamma$ can be expressed as the risk neutral price of a call option on the stock with the same model parameters, e.g. the same volatility, but a greater dividend yield $q+\frac{\gamma \sigma}{\sqrt{T}}$. We achieved this formula by defining the distortion function induced by the distribution function of the standardised asset log-returns. The distribution function to be used depends thus on the choice of the option pricing model. The distribution function of the log-returns must belong to the family of log-concave densities, to guarantee that it induces a distortion function.

In Section 2 we mentioned some desirable properties that distortion functions 

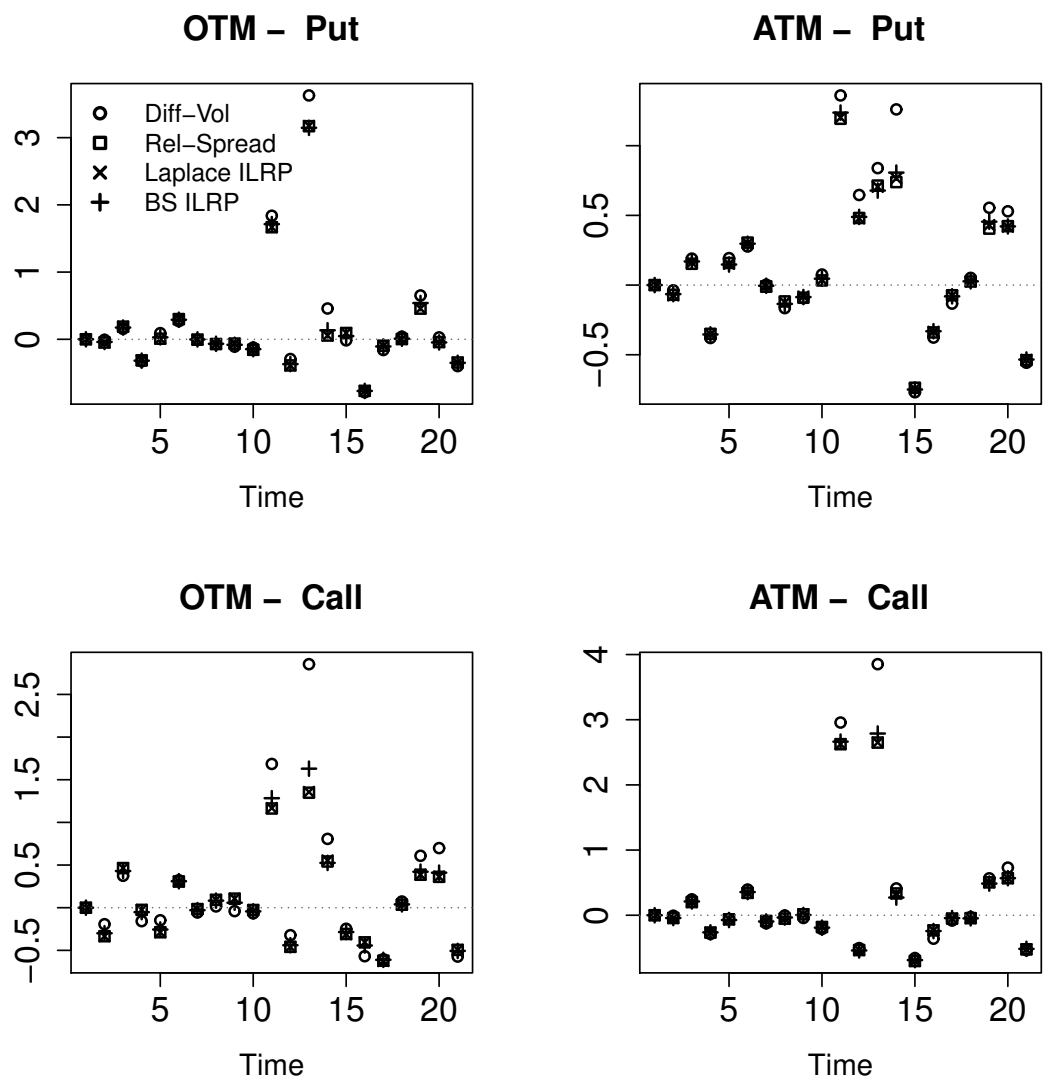

Figure 4: The Figure shows for call and put options with maturities between 0.42 and 0.55 years and moneyness within the two ranges 0.9-0.95 (OTM) and 0.98-1.02 (ATM), the relative difference of four liquidity measures, respectively between two successive timepoints. The liquidity measures are: the relative bidask spread (Rel-Spread), the Black-Scholes and the Laplacian ILRP (BS and Laplace ILRP) and the difference between bid and ask Black-Scholes implied volatilities (Diff-Vol). 
should have, in particular the first derivative of the distortion function should approach infinity at zero and should be equal to zero at 1. In a Black-Scholes setting, we would use the WANG-transform which has all desirable properties. However those properties are not satisfied by the family of distortion functions induced by the Laplace distribution.

Given real market option data, it is possible to compute an implied liquidity risk premium (ILRP), i.e. a tuple of two non-negative numbers $\left(\gamma_{b}, \gamma_{a}\right)$ such that modelled bid and ask prices are exactly equal to real bid and ask prices. This procedure is comparable to the concept of implied volatility. It is then possible to compare bid-ask spreads across different strikes, maturities and underlyings.

Up to now, traders usually describe bid-ask spreads by quoting both bid and ask implied volatilities. There are several advantages using the ILRP instead: it is not always possible to compute an implied bid volatility. The concept of ILRP overcomes this inconsistency. When uncertainty in financial market rises and liquidity dries up, looking only at the difference of Black-Scholes implied bid and ask volatilities often overestimates a change in liquidity by $40 \%$ and more, because the difference of the implied volatilities changes by a higher factor than the relative bid-ask spread. However, the correlation between the relative bid-

ask spread and the ILRP is strong, which makes the ILRP a intuitive measure for liquidity.

\section{References}

[1] H. Albrecher, F. Guillaume and W. Schoutens (2013). Implied liquidity: Model sensitivity. Journal of Empirical Finance, 23, 48-67.

[2] P. Artzner, F. Delbaen, J. M. Eber and D. Heath (1999), Coherent measures of risk, Math. Finance, 9(3), pp. 203-228.

[3] A. Balbás, J. Garrido and S. Mayoral (2009), Properties of distortion risk measures, Methodol. Comput. Appl. Probab., 11, pp. 385-399.

[4] A. E. Bernardo and O. Ledoit (2000), Gain, loss, and asset pricing, J. Polit. Econ., 108(1), pp. 144-172.

[5] P. Carr and D. B. Madan (1999), Option valuation using the fast Fourier transform, J. Comput. Finance, 2(4), pp. 61-73.

[6] A. S. Cherny and D. B. Madan (2009), New measures for performance evaluation, Rev. Financ. Stud., 22(7), pp. 2571-2606.

[7] J. M. Corcuera, F. Guillaume, D. B. Madan and W. Schoutens (2012). Implied liquidity: towards stochastic liquidity modelling and liquidity trading. International Journal of Portfolio Analysis and Management, 1(1), 80-91.

[8] J. M. Corcuera, F. Guillaume, P. Leoni and W. Schoutens (2009), Implied Lévy volatility, Quant. Finance, 9(4), pp. 383-393. 
[9] J. Dhaene, J. Dony, M. B. Forys, D. Linders and W. Schoutens (2012). FIX: The Fear Index-Measuring Market Fear. In Topics in Numerical Methods for Finance (pp. 37-55). Springer, Boston, MA.

[10] H. Föllmer and A. Schied (2011), Stochastic finance: an introduction in discrete time, Walter de Gruyter.

[11] M. Kijima (2006), A multivariate extension of equilibrium pricing transforms: The multivariate Esscher and Wang transforms for pricing financial and insurance risks, Astin Bull., 36(01), pp. 269-283.

[12] M. Kijima and Y. Muromachi (2006), On the Wang transform with fattail distributions, Proceedings of Stanford-Tsukuba Workshop, Stanford University.

[13] M. Kijima and Y. Muromachi (2008), An extension of the Wang transform derived from Bühlmann's economic premium principle for insurance risk, Insurance Math. Econom., 42(3), pp. 887-896.

[14] S. Kusuoka (2001), On Law Invariant Coherent Risk Measures, Adv. Math. Econ., pp. 83-95.

[15] D. B. Madan (2016), Adapted hedging, Ann. Finance, 12(3-4), pp. 305-334.

[16] D. B. Madan and A. Cherny (2010), Markets as a counterparty: an introduction to conic finance, Int. J. Theor. Appl. Finance, 13(08), pp. 11491177.

[17] D. B. Madan and W. Schoutens (2016), Applied Conic Finance, Cambridge University Press.

[18] Tsukahara, H. (2009). One-parameter families of distortion risk measures, Mathematical Finance, 19(4), 691-705.

[19] S. S. Wang (2000), A class of distortion operators for pricing financial and insurance risks, J. Risk Insur., pp. 15-36.

[20] S. S. Wang (2002), A universal framework for pricing financial and insurance risks, Astin Bull., 32(02), pp. 213-234. 